\title{
Opioid use disorder from poppy seed tea successfully treated with buprenorphine in primary care: a case report
}

Scott Hagan ${ }^{1,2^{*}}$ (D), Carol E. Achtmeyer ${ }^{2,3}$, Carly Hood ${ }^{3}$, Eric J. Hawkins $3,4,5$ and Emily C. Williams 4,6

\begin{abstract}
Background: Poppy seeds contain morphine and other opioid alkaloids and are commercially available in the United States. Users of poppy seed tea (PST) can consume several hundred morphine milligram equivalents per day, and opioid dependence from PST use can develop. We report a case of a patient with chronic pain and PST use leading to opioid use disorder (OUD). This case represents the first published report of OUD from PST successfully treated with buprenorphine (BUP) in a primary care setting. The provider in this case used a unique model of care with an opioid prescribing support team to deliver safe and effective care.
\end{abstract}

Case presentation: A 47-year-old man with chronic pain and prescription opioid use presented to primary care to discuss a flare of shoulder pain, and revealed in subsequent conversation a long-standing use of PST to supplement pain control. Attempts at cessation resulted in severe withdrawal symptoms, leading to return to PST use. The primary care provider consulted the VA Puget Sound SUpporting Primary care Providers in Opioid Risk reduction and Treatment (SUPPORT) team to evaluate the patient for OUD. The patient discontinued all opioids, and initiated BUP under the supervision of the primary care provider. He remained on a stable dosage, without relapse, 24 months later.

Conclusions: PST, which can be made through purchase of readily available poppy pods, carries risk for development of OUD and overdose. Herein we highlight the utility of a primary care opioid prescribing support team in empowering a primary care provider to prescribe BUP to treat a patient with complex OUD.

Keywords: Opioid use disorder, Poppy seed tea, Buprenorphine

\section{Background}

Papaver somniferum, also known as opium poppy, is a flowering plant whose seed pods contain numerous opioid alkaloids. Poppy seed pods are commercially available from online and in-store vendors in the United States, but the prevalence of purchase for use in poppy seed tea (PST) concoctions is unknown. The main active opioid alkaloids in PST are morphine, codeine, and thebaine (Table 1). Although the concentration of these substances

\footnotetext{
*Correspondence: scott.hagan1@va.gov

${ }^{2}$ General Medicine Service, Veterans Affairs (VA) Puget Sound Health Care System Seattle Division, Seattle, WA, USA

Full list of author information is available at the end of the article
}

is highly variable depending on the product and the extraction technique [1], users of PST can consume several hundred daily morphine milligram equivalents (MME) [2], increasing risk for opioid overdose and OUD.

Despite legislation enabling primary care providers to prescribe medications for OUD (MOUD) since 2002, treatment for OUD has been difficult to scale in primary care. Consistent evidence suggests that most people diagnosed with OUD never receive any form of treatment [4]. While there are prior case reports of patients with OUD from PST receiving MOUD through addiction treatment programs, there are no known cases of MOUD initiation and maintenance for this indication in a primary care clinic (PCC). Increasing public pressure may soon 
Table 1 Mean opioid concentrations in poppy seed tea

\begin{tabular}{lll}
\hline Opioid Alkaloid & $\begin{array}{l}\text { Concentration }(\mathbf{m g} \\
\text { alkaloid per kg seeds) }\end{array}$ & $\begin{array}{l}\text { Estimated Dose } \mathbf{m g} \\
\text { alkaloid per } 500 \mathbf{~ m L} \\
\text { tea) }\end{array}$ \\
\hline $\begin{array}{l}\text { Morphine } \\
\text { Codeine }\end{array}$ & $352-481$ & $100-136$ \\
Thebaine & $51-68$ & $14-19$ \\
\hline
\end{tabular}

Ranges of mean concentration and estimated dose of 22 different commercially available poppy seed products tested using four different extraction techniques [1]. The estimated dose assumes an average use of $283 \mathrm{~g}$ per $500 \mathrm{~mL}$ [1]

${ }^{a}$ The analgesic or psychoactive properties of thebaine are unknown [3]

lead to increased regulation of the poppy pod industry in the U.S. as it has in other countries [5], and as a result of decreased access, patients using PST may seek evaluations in primary care for OUD-related symptoms.

\section{Case presentation}

A 47-year-old United States veteran with post-traumatic stress disorder (PTSD) and 15-year history of low back, knee, neck, and shoulder pain presented to PCC to discuss pain management. Two weeks prior to the appointment, his primary care provider (PCP) diagnosed a flare of proximal biceps tendinopathy as the cause of worsening right shoulder pain and recommended a course of physical therapy. After reviewing this condition, the patient asked to discuss his opioid contract. He started chronic prescription opioid therapy 12 years ago when other treatments including physical therapy, acetaminophen, piroxicam, gabapentin, and amitriptyline provided insufficient pain relief. Prior opioid regimens included fentanyl, hydrocodone, hydromorphone, morphine, and tramadol. The maximum daily MME was 422 from the combination of a fentanyl patch and hydromorphone tablets prescribed eight years ago in a pain clinic. The following year, pain clinic providers concluded that the patient's undertreated PTSD with accompanying anxiety was significantly exacerbating his pain, and that he was insufficiently engaged in recommended cognitive behavioral therapy for chronic pain. Therefore, they initiated a taper with the goal to discontinue the opioid regimen. However, due to significantly increased pain during the taper, prescriptions for opioids continued at an MME below 90. At the time of the PCC appointment, he had been taking $10 \mathrm{mg}$ three times per day (TID) of oxycodone for two years since establishing care with his current PCP.

During our visit, his pain control was adequate, but he shared, for the first time, that he had been using PST to supplement pain control for the past 7 years. He bought poppy pods at farmer's markets and online retailers and would prepare a hot tea from grounds of the pods.
Depending on his perceived analgesic effect from the tea, he drank 2-4 cups per day, using five large poppy pods per tea serving. The average monthly cost of the PST, which increased substantially in the past 5 years for him, was $\$ 2000$. He did not have psychoactive effects from the tea; however, he did have prolonged withdrawal symptoms when he made attempts to discontinue use. His withdrawal symptoms were shaking chills, intense pain, headache, yawning and diarrhea. These symptoms presented within $24 \mathrm{~h}(\mathrm{~h})$ of abstinence, and would intensify over several days, always leading him to return to PST use. He bought poppy pods through legal sources and never used illicit opioids.

He had been reluctant to report PST use to clinicians for fear of discontinuation of his opioid prescription. In retrospect, his PCP noted that prior annual urine drug screens (UDS) were consistently positive for opiates, which at the PCC's laboratory is a separate assay from oxycodone, indicating that he had consumed another type of opioid (e.g., morphine or codeine from PST). The patient desired complete discontinuation of opioids, both prescription medications and PST, because he was frustrated with opioid dependence for maintenance of an acceptable quality of life, and the financial burden of PST was increasing.

His PCP contacted the VA Puget Sound SUpporting Primary care Providers in Opioid Risk reduction and Treatment (SUPPORT) - a National Center for Patient Safety-funded Patient Safety Center of Inquiry-to assist with evaluation for OUD. One week after the clinic visit, the SUPPORT center social worker assessed the patient and found that he met at least three Diagnostic and Statistical Manual of Mental Disorders, Fifth Edition, criteria for OUD: unsuccessful efforts to cut down use; a great deal of time spent in activities necessary to obtain and use opioids; and the withdrawal symptoms described above following cessation of use. After receiving detailed recommendations for initial dosing and titration from an experienced BUP prescriber through SUPPORT, the PCP prescribed buprenorphine-naloxone (BUP-NLX) for a home initiation 10 days after the initial visit. The patient discontinued all PST and oxycodone usage. Initial withdrawal symptoms started approximately $24 \mathrm{~h}$ later. The starting sublingual (SL) BUP-NLX dose was $2-0.5 \mathrm{mg}$ with option of titration of up to 8-2 mg daily ( $\mathrm{mg} / \mathrm{d}$ ) for withdrawal symptoms. However, the patient continued to experience withdrawal symptoms of sweating, rhinorrhea, restlessness and irritability, after $48 \mathrm{~h}$. These symptoms were only partially relieved with an increase in BUP-NLX dosage to $24-6 \mathrm{mg} / \mathrm{d} 7$ days after initiation. Repeat UDS were positive for BUP, and negative for other opioids. 
10 days after initiation, he no longer experienced opioid cravings on the $24-6 \mathrm{mg} / \mathrm{d}$ dosage. By taking the prescription in doses of 8-2 mg TID, his pain was well controlled throughout the day. However, for the next month, he noticed flushing and anxiety for approximately 30 min after taking each dosage of BUP-NLX. Symptom support with clonidine for flushing and hydroxyzine for anxiety was not effective. Out of concern for intolerance to the BUP-NLX combination product, his PCP trialed BUP monotherapy at the same dose of BUP. Flushing after dosages abated following this medication switch. Because the patient's initial goal was to use BUP to manage withdrawal and subsequently to discontinue all opioids, he requested to taper the dose of BUP to $2 \mathrm{mg}$ TID. Trials of taking lower daily dosing than $6 \mathrm{mg}$ led to increased pain and opioid cravings, so the $2 \mathrm{mg}$ TID dosage of BUP was continued for the next 24 months. Voluntary UDS has only been positive for BUP. The patient felt liberated from the emotional, financial, and physical burden of PST dependence.

\section{Discussion}

Our case highlights an atypical method of opioid use, drinking PST, leading to OUD. Recent studies suggest that PST use is increasing in the United States (U.S.). An analysis of cases of U.S. poppy consumption reported to the National Poison Data Systems and the Food and Drug Administration from 1968 to 2018 reported 64 cases of intentional PST consumption [6]. There was a trend towards increased intentional use of poppy seed products from 2000 to 2018. There are reports of PST use in other countries as well. Among patients surveyed in a New Zealand opioid addiction clinic in 2007, 46\% of patients reported past consumption of PST [7], and among those surveyed in a United Kingdom addiction treatment center in 1990, 93\% of patients reported consumption of PST [8].
The high concentration of morphine in PST leads to risk for overdose and OUD. PST use is directly associated with at least five U.S. fatalities in the twenty-first century [6], and there are six case reports of OUD developing from routine use of PST (Table 2). All cases of OUD were evaluated and treated in addiction treatment centers, and there are no published cases of home initiation of buprenorphine, nor of evaluation and treatment in primary care.

Our patient's prolonged withdrawal symptoms suggest that PST withdrawal may be challenging to manage. Two other case reports of PST withdrawal observed in addiction treatment units described prolonged and severe symptoms $[11,12]$. In addition to the known symptomatology of morphine and codeine withdrawal, discontinuing PST involves clearance of other opioid (thebaine) and non-opioid (papaverine, noscapine) substances whose physiologic effects are poorly understood $[1,3]$.

The patient's improved tolerability to BUP monotherapy is similar to a case [12] in which a patient with PSTrelated OUD better tolerated BUP than the BUP-NLX combination. In that case, the patient's urine was consistently positive for morphine after initiation of BUP-NLX, and subsequently negative while taking BUP, suggesting that precipitated withdrawal while on BUP-NLX may have led to the patient's perception of improved tolerability to monotherapy. There is no evidence in pharmacokinetic studies that BUP-NLX has higher risk of precipitated withdrawal due to naloxone SL absorption compared to BUP $[13,14]$. We had no suspicion of ongoing PST or opioid use while our patient took BUP-NLX given negative urine drug screening. It is possible that our patient had acutely worsened anxiety from withdrawal of either oxycodone or PST, and there was a temporal association with improvement of anxiety 6 weeks into treatment when the switch to BUP monotherapy occurred. In another case report [10], a patient had increased anxiety

Table 2 Case reports of PST-related OUD

\begin{tabular}{|c|c|c|c|c|c|c|}
\hline Patient & Age (years) & Gender & PST Consumption $^{a}$ & Initiation MOUD Dose & Maintenance MOUD Dose & Outcome \\
\hline Case 1 [9] & 37 & M & 14 pods $^{\mathrm{a}} /$ day & Methadone $30 \mathrm{mg} / \mathrm{d}$ & Methadone $30 \mathrm{mg} / \mathrm{d}$ & Not reported \\
\hline Case 2 [2] & 26 & M & $4 \mathrm{~kg}$ seeds/day & Morphine 60 mg BID & Morphine 20 mg BID & Sustained abstinence \\
\hline Case 3 [10] & 43 & $\mathrm{~F}$ & $5-6 \mathrm{~kg}$ seeds/week & BUP $4 \mathrm{mg} / \mathrm{d}$ & BUP $16 \mathrm{mg} / \mathrm{d}$ & Less than monthly use \\
\hline Case 4 [10] & 26 & M & $3 \mathrm{~kg}$ seeds/day & Methadone 30 mg/d & Methadone 110 mg/day & Sustained abstinence \\
\hline Case 5 [11] & 82 & $\mathrm{~F}$ & $1-2$ liters $^{a}$ /day & BUP $0.4 \mathrm{mg} / \mathrm{d}$ & BUP $0.8 \mathrm{mg} / \mathrm{d}$ & Sustained abstinence \\
\hline Case 6 [12] & 42 & M & Unknown ${ }^{a}$ & BUP-NLX $16-2 \mathrm{mg} / \mathrm{d}$ & BUP $18 \mathrm{mg} / \mathrm{d}$ & Sustained abstinence \\
\hline Case $7^{b}$ & 47 & M & 2-4 cups/day & BUP-NLX 8-2 mg/d & BUP $6 \mathrm{mg} / \mathrm{d}$ & Sustained abstinence \\
\hline
\end{tabular}

Summary of existing case reports of PST-related OUD highlighting consumption, treatment with MOUD, and outcomes

F, female; M, male; BID, twice a day

${ }^{a}$ Did not quantify amount of poppy seeds used

${ }^{\mathrm{b}}$ Case 7 is the patient described in this report 
when he discontinued PST and started methadone. Providers prescribed venlafaxine prescription for control of symptoms. Monitoring for the effects of opioid abstinence on mental health, even while patients take MOUD, is important to help patients to engage in treatment.

The misinterpretation of the patient's prior UDS results, specifically that a positive result for opiates was thought by clinicians to be due to the patient's oxycodone use, was a missed opportunity to identify the patient's PST use at an earlier point in his care. Prior research suggests that many providers who order UDS have limited understanding of opioid metabolism and the cross-reactivity of immunoassays, and misinterpretation of urine drug tests is common [15]. Expansion of UDS interpretation support programs [16] may help to improve provider interpretation of complicated UDS results for opioids.

There are many parallels between PST and kratom, an herbal supplement derived from tree leaves containing alkaloids that are opioid receptor agonists. Like PST, kratom is commercially available from online and instore vendors, and is not regulated by the FDA. Consumption of kratom through brewed teas, powders, or pills produces analgesic effects, and there are increasing cases of OUD developing from routine use, as well as cases of individuals using kratom as treatment for OUD from other opioids. With an estimated $0.7 \%$ annual use in the U.S. adult population [17], kratom is likely more widely consumed than PST in the U.S., but treatment guidelines for OUD resulting from either substance do not exist. Due to uncertainties in the relative strength of opioids consumed from these substances, it may be difficult for the treating clinician to determine a sufficient dosage for initiation of BUP, which may explain the varied BUP initiation doses reported in Table 2. There may be uncertainty around maintenance doses as well, as prior case reports indicate varied BUP maintenance dosing. In a recent analysis of patients with OUD from kratom [18], those with higher self-reported kratom use at baseline tended to require a higher maintenance dose of BUP to manage OUD. Our patient initially needed a high dosage of BUP to manage withdrawal symptoms, but was later able to tolerate $6 \mathrm{mg}$ daily during sustained remission. Symptom-directed titration of BUP, along with short interval follow-up, were the keys to successful dosing in our case. Given the variability in initiation and maintenance dosages, it is important that withdrawal symptoms are monitored closely with results used to inform adjustments to BUP dosing.

This case encapsulates the important progress being made for the treatment of patients with OUD in the primary care setting. Historically, patients with OUD could only access MOUD through federally regulated Opioid Treatment Programs. With the substantial increase in
OUD largely a result of increased U.S. opioid prescribing in the past several decades [19], these facilities have been unable to keep pace with the high demand for MOUD [20]. Congress passed the Drug Addiction Treatment Act of 2000 (DATA 2000), which allowed qualified providers to prescribe BUP and naltrexone across various healthcare settings, including primary care [21]. These medications effectively treat OUD by reducing or eliminating illicit substance use and lowering risk of fatal and non-fatal overdose, and are safe medications [22] for prescribing by a general practitioner. A shift that further facilitated prescribing in primary care was the introduction of home initiation of BUP [23]. Therefore, a variety of care models now exist to encourage PCPs to diagnose and to treat OUD [24].

The SUPPORT center at the VA Puget Sound Medical Center, funded by the Veterans Health Administration's National Center for Patient Safety [25], is a unique model of care which offers PCPs a range of options for prescribing MOUD: (1) open-access consultation with a social worker specializing in OUD and an experienced BUP provider, who give recommendations to help the PCP to diagnose OUD and to initiate BUP independently; (2) initial diagnosis and treatment with BUP by the SUPPORT team, with transition of prescription to the PCP when the BUP dosage has stabilized; and (3) initiation and indefinite prescribing of BUP by the SUPPORT team if the PCP feels uncomfortable with managing BUP.

Although recently waivered by the Drug Enforcement Agency to prescribe BUP, the PCP in this case had never prescribed MOUD, and yet felt empowered to initiate BUP-NLX using the consultation model described above. The long-term relationship that existed between the PCP and the patient, the availability of help from the SUPPORT team, and the option to take BUP through PCC without the burden and stigma of an addiction treatment program were important components to success. Our model of care allows PCPs with limited experience with MOUD to manage OUD in primary care.

\section{Conclusion}

PST, which can be made through purchase of readily available poppy pods, carries risk for development of OUD and overdose. Policymakers should consider interventions to regulate the sale of these products. Primary care clinicians, with the help of office-based opioid therapy support teams, can be empowered to identify and treat OUD with BUP in primary care.

\section{Abbreviations}

PST: Poppy seed tea; OUD: Opioid use disorder; MOUD: Medications for opioid use disorder; SUPPORT: SUpporting Primary care Providers in Opioid Risk 
reduction and Treatment; MME: Morphine milligram equivalents; PCC: Primary care clinic; PTSD: Post-traumatic stress disorder; TID: three times a day; mg/d: Milligrams per day; $\mathrm{h}$ : Hours; UDS: urine drug screen; PCP: Primary care provider; BUP-NLX: Buprenorphine-naloxone; BUP: Buprenorphine; SL: Sublingual ; BID: twice a day.

\section{Acknowledgements}

Not applicable.

\section{Authors' contributions}

SH drafted the manuscript and served as the PCP for this patient. CA and $\mathrm{CH}$ provided consultation for the case as members of the SUPPORT team and were major contributors to the manuscript. EH and EW provided critical feedback on the manuscript from their perspective as substance use disorder researchers. All authors read and approved the final manuscript.

\section{Author's information}

Dr. Scott Hagan is a primary care physician, clinician educator, and Medical Director of the Seattle VA Primary Care Clinic. His clinical interests include evidence-based treatment of adults with substance use disorders, obesity medicine, and diagnostic reasoning.

\section{Funding}

No funding was provided for the drafting of this manuscript.

\section{Availability of data and materials}

No data was collected for the purposes of this study. The information used for this case report comes from the patient's record.

\section{Declarations}

\section{Ethics approval and consent to participate}

The patient described in this case report has provided written consent for this information to be submitted for publication. The manuscript does not feature patient identifiers and protects the patient's anonymity. As a case report, Ethics Committee review is not indicated.

\section{Consent for publication}

The patient described in this case report has provided written consent for this information to be submitted for publication. A copy of this written consent is available if needed.

\section{Competing interests}

The authors have no competing interests.

\section{Author details}

'Department of Medicine, University of Washington School of Medicine, Seattle, WA, USA. ${ }^{2}$ General Medicine Service, Veterans Affairs (VA) Puget Sound Health Care System Seattle Division, Seattle, WA, USA. ${ }^{3}$ Center of Excellence in Substance Addiction Treatment and Education, Veterans Affairs (VA) Puget Sound Health Care System Seattle Division (S116ATC), 1660 S. Columbian Way, Seattle, WA, USA. ${ }^{4}$ Health Services Research \& Development (HSR\&D), Seattle Center of Innovation for Veteran-Centered and Value-Driven Care, Veterans Affairs (VA) Puget Sound Health Care System, Seattle, WA, USA. ${ }^{5}$ Department of Psychiatry and Behavioral Sciences, University of Washington School of Medicine, Seattle, WA, USA. ${ }^{6}$ Department of Health Systems and Population Health, University of Washington School of Public Health, Seattle, WA, USA.

Received: 19 May 2021 Accepted: 16 November 2021

Published online: 03 December 2021

\section{References}

1. Powers D, Erickson S, Swortwood MJ. Quantification of morphine, codeine, and thebaine in home-brewed poppy seed tea by LC-MS/MS. J Forensic Sci. 2018;63(4):1229-35.

2. King MA, Mcdonough MA, Drummer OH, Berkovic SF. Poppy tea and the baker's first seizure. Lancet. 1997;350(9079):716.
3. Eisenreich A, Sachse B, Gürtler R, Dusemund B, Lindtner O, Schäfer B. What do we know about health risks related to thebaine in food? Food Chem. 2020;309: 125564

4. Mental Health Services Administration. National survey of substance abuse treatment services (N-SSATS). Substance Abuse and Mental Health Services Administration. 2018.

5. Lurie P, Macleery L, Sorscher S. Letter to the surgeon general re: contaminated "unwashed" poppy seeds. Center for Science in the Public Interest. June 3, 2019

6. Greenthal E, Lurie P, Doyon S. Opioid exposure associated with poppy consumption reported to poison control centers and the U.S. food and drug administration. Clin Toxicol. 2021;59(8):746-55.

7. Braye K, Harwood T, Inder R, Beasley R, Robinson G. Poppy seed tea and opiate abuse in New Zealand. Drug Alcohol Rev. 2007;26(2):215-9.

8. London M, O'regan T, Aust P, Stockford A. Poppy tea drinking in East Anglia. Br J Addict. 1990;85(10):1345-7.

9. Unnithan S, Strang J. Poppy tea dependence. Br J Psychiatry. 1993;163:813-4.

10. Lloyd-jones DM, Bonomo Y. Unusual presentations for pharmacotherapypoppy seed dependence. Drug Alcohol Rev. 2006;25(4):375-6.

11. Nanjayya SB, Murthy P, Chand PK, et al. A case of poppy tea dependence in an octogenarian lady. Drug Alcohol Rev. 2010;29(2):216-8.

12. Haber I, Pergolizzi J, Lequang JA. Poppy seed tea: a short review and case study. Pain Ther. 2019;8(1):151-5.

13. Harris DS, Jones RT, Welm S, Upton RA, Lin E, Mendelson J. Buprenorphine and naloxone co-administration in opiate-dependent patients stabilized on sublingual buprenorphine. Drug Alcohol Depend. 2000;61(1):85-94.

14. Strain EC, Moody DE, Stoller KB, Walsh SL, Bigelow GE. Relative bioavailability of different buprenorphine formulations under chronic dosing conditions. Drug Alcohol Depend. 2004;74(1):37-43.

15. Chua I, Petrides AK, Schiff GD, et al. Provider misinterpretation, documentation, and follow-up of definitive urine drug testing results. J Gen Intern Med. 2020;35(1):283-90.

16. Stammet MM, Spradley SS. Evaluation of treatment changes following electronic consultation to a pharmacist-run urine drug testing service in a veterans healthcare system. J Opioid Manag. 2016;12(6):389-95.

17. Palamar JJ. Past-year kratom use in the U.S.: estimates from a nationally representative sample. Am J Prev Med. 2021;61(2):240-5.

18. Weiss ST, Douglas HE. Treatment of kratom withdrawal and dependence with buprenorphine/naloxone: a case series and systematic literature review. J Addict Med. 2021;15(2):167-72.

19. Rudd RA, Aleshire N, Zibbell JE, Matthew Gladden R. Increases in drug and opioid overdose deaths_-United States, 2000-2014. Am J Transplant. 2016;16(4):1323-7.

20. Jones CM, Campopiano M, Baldwin G, McCance-Katz E. National and state treatment need and capacity for opioid agonist medication-assisted treatment. Am J Public Health. 2015;105(8):e55-63.

21. Substance Abuse and Mental Health Services Administration. Medications for opioid use disorder. Treatment improvement protocol (TIP) series 63, full document. 2018

22. Sordo L, Barrio G, Bravo MJ, et al. Mortality risk during and after opioid substitution treatment: systematic review and meta-analysis of cohort studies. BMJ. 2017;357: j1550.

23. Martin SA, Chiodo LM, Bosse JD, Wilson A. The next stage of buprenorphine care for opioid use disorder. Ann Intern Med. 2019;170(11):821-2.

24. Korthuis PT, McCarty D, Weimer M, Bougatsos C, Blazina I, Zakher B, et al. Primary care-based models for the treatment of opioid use disorder: a scoping review. Ann Intern Med. 2017;166(4):268-78.

25. Williams EC, Hawkins EJ."Increasing Safety for Veteran Patients at High Risk of Adverse Opioid-Related Outcomes," a Patient Safety Center of Inquiry funded by VA's National Center for Patient Safety.

\section{Publisher's Note}

Springer Nature remains neutral with regard to jurisdictional claims in published maps and institutional affiliations. 